\title{
¿QUÉ OPINA EL ALUMNADO DE PRIMARIA SOBRE EL USO DE RUTINAS DEL PENSAMIENTO COMO INSTRUMENTO DE EVALUACIÓN FORMATIVA?
}

What is the primary school students' opinion about the use of thinking routines as an instrument of formative evaluation?

Qual é a opinião dos alunos do ensino primário sobre a utilização de rotinas de pensamento como instrumento de avaliação formativa?

\section{César Caballero San José (1)}

Noelia García-Martín (2)

Ruth Pinedo González (3)

Manuel Cañas Encinas (4)

\author{
(1) Universidad de Valladolid. España. Correo electrónico: cescaba@hotmail.com \\ (2) Universidad de Valladolid. España. Correo electrónico: noeliagarcia125@gmail.com \\ (3) Universidad de Valladolid. España. Correo electrónico: ruth.pinedo@uva.es \\ (4) Universidad de Valladolid. España. Correo electrónico: manubernardos97@gmail.com
}

\section{Resumen}

Las metodologías de enseñanza denominadas activas necesitan de una evaluación congruente con el nuevo rol que se asigna al alumnado en su proceso de aprendizaje. El objetivo de esta experiencia educativa es analizar las opiniones del alumnado respecto al uso de un sistema de evaluación formativa y compartida mediante rutinas del pensamiento para evaluar un proyecto de aprendizaje, ya que es la primera vez que se lleva a cabo en el aula. Para ello, se ha seguido la metodología cualitativa y un diseño de estudio de caso. Los participantes han sido 20 estudiantes de $6^{\circ}$ curso de Educación Primaria. Los resultados revelan que los estudiantes prefieren la evaluación formativa y compartida y consideran que es un proceso más justo y aprenden más. En conclusión, la evaluación formativa y compartida mediante rutinas de pensamiento es valorada por el alumnado de forma muy positiva.

Palabras clave: Evaluación formativa y compartida; Educación Primaria; rutinas de pensamiento. 


\begin{abstract}
Active teaching methodologies need an evaluation system that is congruent with the new role assigned to students in their learning process. The objective of this educational experience is to analyze students' opinions regarding the use of a system of formative and shared assessment using thinking routines to evaluate a learning project, since this is the first time that it is carried out in the classroom. For this, the qualitative methodology and a case study design have been followed. The participants were 20 students from the 6th year of Primary Education. The results reveal that the students prefer formative and shared assessment and consider that it is a fairer process. In conclusion, the formative and shared assessment using thinking routines is valued by the students in a very positive way.
\end{abstract}

Keywords: Formative and shared assessment; Primary Education; thinking routines

\title{
Resumo
}

As metodologias de ensino ativas necessitam de uma avaliação que seja congruente com o novo papel atribuído aos alunos no seu processo de aprendizagem. O objetivo desta experiência educativa é analisar as opiniões dos alunos sobre o uso de um sistema de avaliação formativa e partilhada, por meio de rotinas de pensamento, para avaliar um projeto de aprendizagem, uma vez que é a primeira vez que ele é realizado na aula. Deste modo, usou-se uma metodologia qualitativa, através dode um estudo de caso. Participaram no estudo 20 alunos do $6^{\circ}$ ano do Ensino Básico. Os resultados revelam que os alunos preferem a avaliação formativa e partilhada, considerando que é um processo mais justo e no qual aprendem mais. Em suma, a avaliação formativa e partilhada, através de rotinas de pensamento é valorada pelos alunos de uma forma muito positiva.

Palavras-chave: Avaliação formativa e partilhada; Educação Primária; rotinas de pensamento

\section{Introducción}

La necesidad de preparar al alumnado para las exigencias del siglo XXI nos lleva a repensar el modelo de escuela y, con él, el modelo de evaluación que los docentes emplean (Johnson \& Johnson, 2014). Los procesos de evaluación deben ir en esta 
misma línea para mantener la coherencia entre todos los elementos curriculares. Así nace la evaluación formativa y compartida, con el fin de mejorar los procesos de enseñanza-aprendizaje, la práctica docente y la mejora continua, consciente y reflexiva del aprendizaje del alumnado (Pérez-Pueyo, 2013).

Estos sistemas evaluativos no se pueden llevar a cabo sin una metodología coherente con los mismos y las denominadas metodologías activas posibilitan tal proceso (Labrador \& Andreu, 2008). Una de las metodologías activas más utilizada es el Aprendizaje Basado en Proyectos (ABP) en la que los estudiantes desarrollan diversas estrategias organizativas de acuerdo con sus ritmos de aprendizaje, pues el trabajo autónomo de investigación para crear un producto final grupal es la clave de esta metodología (Vergara, 2016).

Por último, se debe mencionar el Pensamiento Visible, un enfoque basado en visibilizar y documentar los procesos de pensamiento del alumnado para apoyar el desarrollo de la comprensión y el aprendizaje. Un aspecto fundamental dentro de este enfoque son las denominadas rutinas del pensamiento, las cuales se basan en organizadores gráficos sencillos que ayudan a los estudiantes a presentar ideas, organizarlas o profundizar en ellas, es decir, a reflexionar sobre su aprendizaje.

Por tanto, el objetivo de este estudio es analizar las opiniones que el alumnado tiene sobre el uso de rutinas de pensamiento como herramienta para realizar una evaluación formativa.

\section{Método}

Las rutinas de pensamiento se convierten en herramientas ideales para llevar a cabo un sistema de evaluación formativa y compartida porque en ambos casos se busca el aprendizaje y la reflexión. Por ello se llevó a cabo un proyecto de aprendizaje denominado "I Concurso de Poesía del CEIP El Abrojo" en el que se diseñó, durante dos sesiones y de forma cooperativa, entre alumnado y profesorado, un sistema de evaluación formativa y compartida en el que se utilizaron como instrumentos las rutinas de pensamiento. El proyecto se realizó durante dos meses y se llevaron a cabo 7 actividades formativas diferentes, tras las cuales cada grupo de trabajo realizó la rutina del pensamiento "El semáforo" decidiendo qué cosas han hecho bien, qué cosas pueden mejorar y qué cosas creían que estaban mal. Además, se finalizó el proyecto con la rutina individual la "Escalera de la Metacognición” donde cada participante reflexionó 
sobre su aprendizaje. Posteriormente se analizaron las opiniones del alumnado participante sobre todo el proceso, que son los datos que se exponen a continuación.

Para analizar las opiniones del alumnado participante se ha seguido un diseño de estudio de caso. Como técnicas de recogida de datos se han empleado un cuestionario con preguntas cerradas de opción múltiple y la entrevista en profundidad. Los participantes han sido 20 estudiantes de $6^{\circ}$ curso de Educación Primaria.

\section{Resultados y discusión}

Como vemos en la Figura 1, la mayoría de los participantes prefiere la evaluación formativa. Al analizar sus razones podemos destacar:

- La evaluación formativa me parece buena porque podemos aprender de los errores de los demás y de los de nosotros mismos (Entrevista2).

- Que nos damos las razones de la nota (Cuestionario).

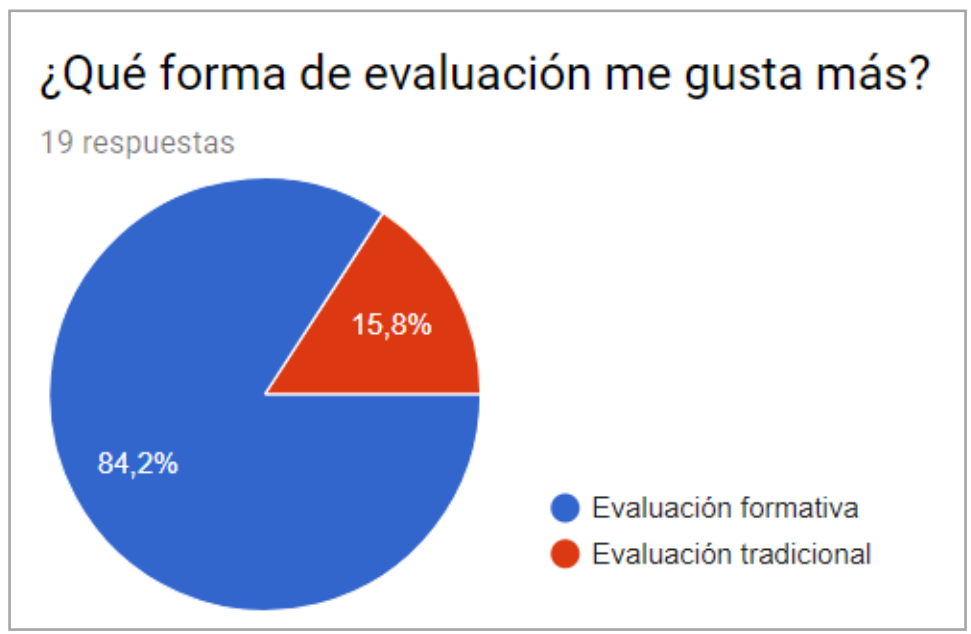

Figura 1. Elección del alumnado entre evaluación tradicional y evaluación formativa

En la Figura 2 podemos ver que los participantes prefieren la coevaluación frente a la auto y heteroevaluación. Al analizar las razones podemos destacar:

- Porque nos podemos poner de acuerdo en la nota y veo lo que opina el compañero que tiene que saber más o menos lo mismo que yo (Entrevista1).

- Aprendemos más de ver cómo realizan las actividades los otros grupos desde varios puntos de vista (Cuestionario). 


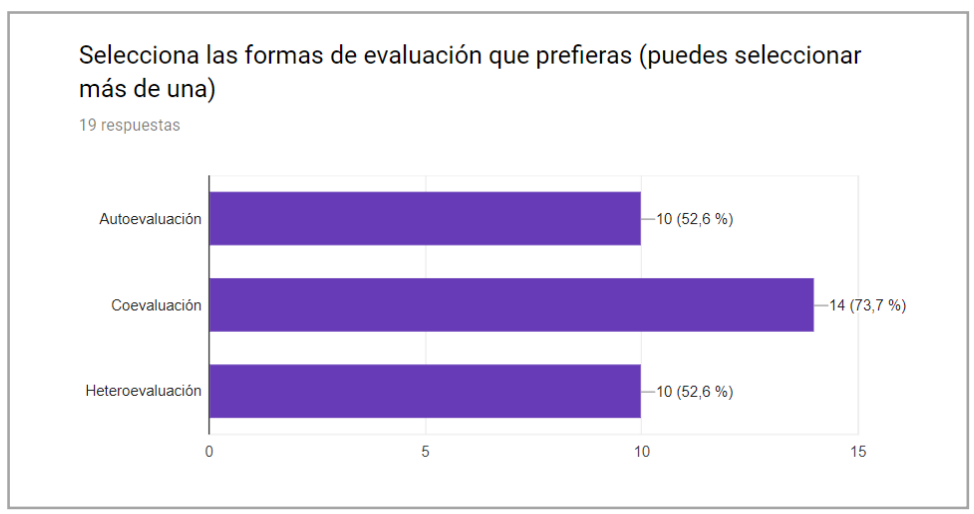

Figura 2. Formas de evaluación seleccionadas por el alumnado

En la Figura 3 se puede ver que la mayoría del alumnado de primaria considera que con evaluación formativa aprenden más. Si atendemos a sus razones se puede destacar:

- Lo que más me aporta es el aprendizaje, porque podemos, por ejemplo, cuando nos dan un examen que no es el nuestro y lo corregimos, podemos aprender de los errores de los demás y pues aprendemos aún más. También el feed-back de los compañeros y el profesor, te sirve para aprender (Entrevista2).

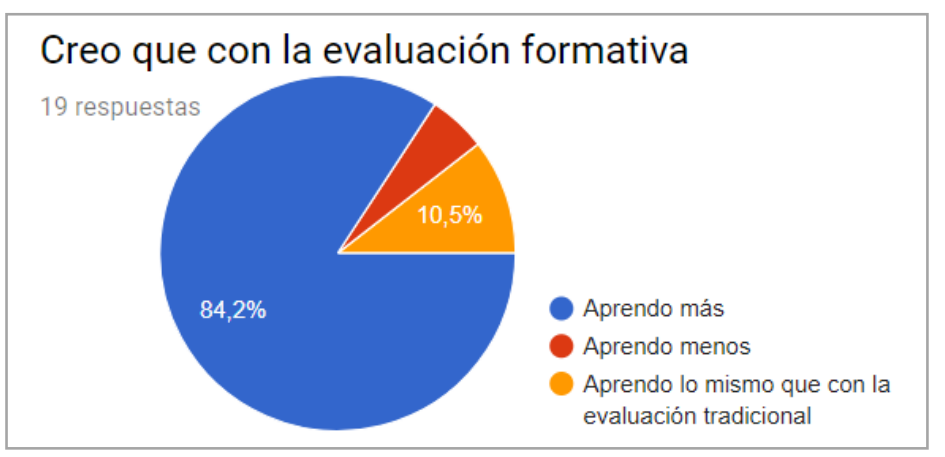

Figura 3. Concepciones sobre la evaluación formativa y el aprendizaje

En la Figura 4 se puede observar que la gran mayoría de los participantes consideran de gran interés poder participar en el diseño de los instrumentos de evaluación que se van a usar. Si atendemos a sus razones podemos destacar:

- Me parece bien, porque si fallas, por ejemplo, no puedes poner de excusa que se evalúa de tal manera porque lo has elegido tú (Entrevista2).

- Me ha gustado diseñar los instrumentos porque podíamos elegir entre los que más nos gusten y elegir entre la autoevaluación, coevaluación y heteroevaluación (Entrevista1). 


\section{¿Te ha gustado participar en el diseño de los instrumentos de evaluación del proyecto?}

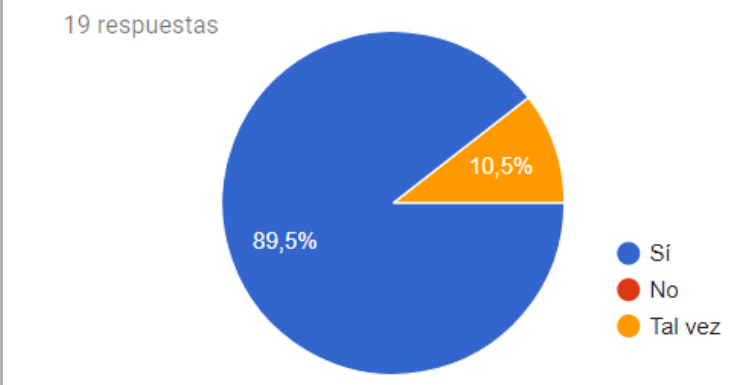

Figura 4. Interés por participar en el diseño de los instrumentos de evaluación.

\section{Conclusiones}

La evaluación formativa y compartida utilizando rutinas de pensamiento es valorada por el alumnado de forma muy positiva, pues destacan que aprenden más y es un proceso más justo. Además, las rutinas de pensamiento permiten desarrollar hábitos reflexivos que generan aprendizajes profundos, convirtiendo la evaluación en parte importante del proceso de enseñanza-aprendizaje.

\section{Referencias}

Johnson, D., \& Johnson, R. (2014). La evaluación en el aprendizaje cooperativo. Cómo mejorar la evaluación individual a través del grupo. Madrid, España: SM.

Labrador, M. J., \& Andreu, M. A. (2008). Metodologías activas. Universidad Politécnica de Valencia. Valencia: Editorial de la UPV. https://doi.org/V-46422008

Płrez-Pueyo, A. (2013). Programar y evaluar competencias b«sicas en 15 pasos. Barcelona, España: Gra\%o

Ritchhart, R., Church, M., \& Morrison, K. (2014). Hacer visible el pensamiento: cómo promover el compromiso, la comprensión y la autonomía de los estudiantes. Barcelona, España: Paidós.

Vergara, J. J. (2016). Aprendo porque quiero. El aprendizaje basado en proyectos paso a paso. Madrid, España: SM. 